\title{
Nature of hematopoietic system alteration and organ damage induced by fungal filtrate of Pestalotiopsis microspora in vivo
}

\author{
Taofeeq Garuba ${ }^{1}$, Adebola Azeez Lateef ${ }^{1}$, Soliu Abiola Atunwa², Abibat Yetunde Lateef ${ }^{1}$, Olajumoke Oluwabunmi Kolawole ${ }^{1}$, \\ Saheed Sabiu ${ }^{3 *}$ \\ ${ }^{1}$ Department of Plant Biology, University of Ilorin, Ilorin, Nigeria. \\ ${ }^{2}$ Department of Pharmacology and Toxicology, University of Ilorin, Ilorin, Nigeria. \\ ${ }^{3}$ Department of Biotechnology and Food Science, Faculty of Applied Sciences, Durban University of Technology, Durban, South Africa.
}

\begin{tabular}{l}
\hline ARTICLE INFO \\
\hline Received on: $11 / 08 / 2020$ \\
Accepted on: $18 / 11 / 2020$ \\
Available online: $05 / 03 / 2021$ \\
\\
\hline Key words: \\
Ascomycetes, endophytes, \\
organ damage, Pestalotiopsis \\
microspora, selective toxicity.
\end{tabular}

\section{INTRODUCTION}

For years, studies have demonstrated the diversity and richness of the plant kingdom in valuable bioactive substances of therapeutic importance against human diseases (Chin et al., 2006; Olumese and Oboh, 2016; Radhia et al., 2018). Specifically, the mushrooms including certain species of Pestalotiopsis have been reported as an excellent source of nutrients and therapeutic compounds for both animals and humans (Garuba et al., 2018).

Pestalotiopsis is a fungus belonging to ascomycetes. The species are widely distributed (Wei and $\mathrm{Xu}, 2004$ ) and studies are emerging on their ability to incite disease development and their

\section{"Corresponding Author}

Saheed Sabiu, Department of Biotechnology and Food Science, Faculty of Applied Sciences, Durban University of Technology, Durban, South Africa. E-mail: saeed.sabiu@gmail.com endophytic nature (Maharachchikumbura et al., 2011). This group of fungi is responsible for many diseases in plants such as leaf streak disease, leaf spot, and grape decay caused by Pestalotiopsis royenae, Pestalotiopsis heterocornis, and Pestalotiopsis menezesiana, respectively. Pestalotiopsis palmarum causes leaf spot, petiole blights, and bud rots in palms such as date palm, coconuts, and oil palm (Elliot, 2018). Pestalotiopsis longiseta and Pestalotiopsis theae that caused tea gray blight disease produce oxysporone that is phytotoxic (Nagata and Ando, 1989). Pestalopyrone, pestalotin, hydroxy-pestalotin, and oxysporone are secreted by Pestalotiopsis oenotherae that is responsible for leaf spot disease of evening primrose (Venkatasubbaiah et al., 1991). Despite their disease-causing potential, many of them including Pestalotiopsis microspora, which is usually saprophytic but endophytic in many plant species (Metz et al., 2000), produce bioactive compounds that are medicinally important. For instance, isoxazoline, indolizine, pestacin, isopestacin, yadanzioside D, and heptanol have been isolated from P. microspora and they 
are pharmaceutically useful in drug production against several diseases (Garuba et al., 2018; Joseph and Priya, 2011). Deshmukh et al. (2017) have also recognized this group of fungi as a prolific producer of beneficial secondary metabolites. While the fungus is industrially and pharmaceutically important as an efficient decomposer of wood, plastics, leather, and a reliable source of an anticancerous agent, taxol (Tiwari, 2015), it could also produce toxic metabolites that are noxious to body organs (Sharma et al., 2016).

Although studies by Thikare et al. (2011) and Vinay et al. (2014) have, for instance, demonstrated and established toxicity profiles of culture filtrates from Rhizopus oryzae and Fusarium semitectum, respectively, continuous toxicological assessment of fungi to improve on the currently available limited data is imperative to elucidate their safety applications in animals and human. This is also the case with P. microscopora, where despite its economic and therapeutic significance there are no scientific reports on its safety profiles on the systemic organs of animals feeding on the parent plants from where it is normally isolated. Hence, for the first time, this study investigated the toxicological implications of the culture filtrate of P. microspora on the hematological indices and vital metabolic organs of Wistar rats.

\section{MATERIALS AND METHODS}

\section{Collection and authentication of $\boldsymbol{P}$. microspora}

Pestalotiopsis microspora was collected from the stock in Plant Pathology Laboratory of the Department of Plant Biology, University of Ilorin, Ilorin, Nigeria. The identity of the fungus had been previously authenticated using both morphological and molecular methods (Garuba et al., 2018) and kept under the voucher specimen number UIL/MYCOLOGY2018/PM003.

\section{Preparation of $\boldsymbol{P}$. microspora filtrate (PMF)}

Adopting the method of Garuba et al. (2013), the culture filtrate of the fungi was prepared. Briefly, a $5 \mathrm{~mm}$ of agar disk from a 7-day-old culture of $P$. microspora was inoculated into potato dextrose broth (PDB) and incubated at $25^{\circ} \mathrm{C} \pm 2{ }^{\circ} \mathrm{C}$ for 14 days. The culture filtrate was filtered into presterilized conical flasks using Whatman no. 1 filter paper. The residue (fragments of mycelia) was discarded and the filtrate was stored in a refrigerator at $4^{\circ} \mathrm{C} \pm 2{ }^{\circ} \mathrm{C}$ (Jalander and Gachande, 2012).

\section{Animals used and the experimental design}

The study was reviewed and approved (UERC/ ASN/2019/1878) by the Animal Ethical Committee of the University of Ilorin, Ilorin, Nigeria, adopting the Guidelines of National Research Council Guide for the Care and Use of Laboratory Animals [National Research Council (NRC), 2011] and principles of Good Laboratory Procedures (World Health Organization, 1998). Healthy male Wistar rats $(n=30)$ weighing $120 \pm 10 \mathrm{~g}$, procured from the Central Animal House, University of Ilorin, Ilorin, were used in this study. The animals were kept in a well-aerated room (temperature $25^{\circ} \mathrm{C} \pm 2{ }^{\circ} \mathrm{C}, 12$ hours alternating photoperiods, and $45 \%-50 \%$ relative humidity) and maintained on normal rat feed (Vita Feed ${ }^{\mathrm{R}}$, Nigeria) and potable water ad libitum. After 7 days of acclimatization, the animals were separated into two groups for acute $(n=10)$ and repeated dose $(n=20)$ toxicity testing, respectively.

For the acute toxicity study, the method of Thikare et al. (2011) and Organization of Economic Co-operation and Development (OECD) guideline 420 [Organization of Economic Co-operation and Development (OECD), 2001] were adopted with slight modification. Briefly, the rats that were earlier fasted for 18 hours were randomly divided into two groups ( $n=5$ per cage). Rats in group 1 were orally given $2 \mathrm{ml}$ PDB and served as a control, while group 2 animals were administered a single oral dose $(2 \mathrm{ml} /$ $\mathrm{kg}$ b.w.) of PMF. Subsequently, the animals were placed under close monitoring within the first 4-12 hours and then 24 hourly for 14 days. The rats were weighed before the commencement of the experiment (day 0) and, respectively, on days 3, 7, 10, and 14 with attention paid to any notable changes in behavior and occurrence of diarrhea, depression, weakness and fatigue, sedation, salivation, lethargy, and so on (Sabiu et al., 2015).

In the 28-day repeated dose toxicity testing, the animals were randomly divided into four groups of five rats each as per OECD guideline 408 [Organization of Economic Co-operation and Development (OECD), 2006]. For the dose administration, the control group was administered $2 \mathrm{ml}$ PDB and designated as group 1 , while groups 2,3 , and 4 were given $0.25,0.5$, and 0.75 $\mathrm{ml} / \mathrm{kg}$ b.w. of PMF, respectively, for 28 days following previously described methods (Thikare et al., 2011; Vinay et al., 2014) with slight modifications. All administrations were carried out once daily via oral gavage, while records of the weight of the rats were taken weekly with daily observation on possible signs of humane endpoint, morbidity, and behavioral changes (Sabiu et al., 2015). The observation on the weight changes was used to compute possible weight gain (\%) as the relative weight of the animals at the start and end of the experiment, respectively (Sabiu et al., 2015).

On the 15th and 29th days for the acute and repeated dose testing periods, respectively, the rats were humanely euthanized under halothane $(1.5 \%)$ anaesthetization and blood was collected from the jugular vein into ethylene diamine tetraacetic acid vacutainer tubes. The tubes were then centrifuged $(15,000 \mathrm{rpm}, 10$ minutes) and used for hematological analysis. Organs including the liver, heart, and kidneys were immediately excised subsequent to dissection and thereafter preserved (10\% buffered formalin solution) for histopathological examinations.

\section{Hematological analysis}

Using an Automated Hematological Analyzer (Sysmex KX21, Japan), the hematological indices [white blood corpuscle (WBC), neutrophils (NEUT), lymphocytes (LYMPH), basophils (BASO), red blood corpuscle (RBC), hemoglobin (HGB), packed cell volume $(\mathrm{PCV})$, mean corpuscular volume (MCV), mean corpuscular hemoglobin $(\mathrm{MCH})$, mean corpuscular hemoglobin concentration (MCHC), and platelet (PLT)] were determined.

\section{Histopathological analysis}

This was carried out at the Histopathology Laboratory, Faculty of Veterinary Medicine, University of Ilorin, Nigeria, using the method of Bing and Edward (2015). Briefly, the representative sections of the preserved organs (liver, heart, and kidneys) were successively dehydrated in graded ethanol $(70 \%$ 
$100 \%)$. This was followed by the replacement of the ethanol with an appropriate solvent that is readily mixable with the embedding medium in preparation for embedding. Subsequently, the tissue sections of the organs were treated with xylene to make them transparent (clearing) prior to embedding in an oven maintained at $58^{\circ} \mathrm{C}-60^{\circ} \mathrm{C}$. This allowed for solvent evaporation and provision of spaces within the tissues for para-n. Exactly, $5 \mu \mathrm{m}$ from each paraffin-impregnated tissue was floated on water and then transferred to a glass slide for staining (hematoxylin and eosin) and subsequent viewing (with a simple Olympus light microscope) with magnification of $\times 10$.

\section{Data analysis}

The data were subjected to descriptive statistics and tests for significant difference were carried out by one-way analysis of variance complemented with Duncan's test using the Statistical Package for Social Science (SPSS) for Windows, version 16.0 (SPSS Inc., Chicago, IL). The results are expressed as the mean \pm standard error of the mean (SEM) with $p$-values of $<0.05$ considered statistically significant.

\section{RESULTS}

\section{Acute toxicity}

The acute oral administration of $2 \mathrm{ml} / \mathrm{kg}$ b.w. of PMF produced no observable clinical sign or behavioral changes in Wistar rats. The data on weight changes of the animals at days 0,3 , 7,10 , and 14 revealed no significant difference $(p>0.05)$ between the PMF-treated rats and the animals in the control group with an overall weight gain of $14.23 \%$ and $17.44 \%$, respectively (Fig. 1). Similarly, no statistically significant variation $(p>0.05)$ was observed in the hematological parameters investigated between the control group and the PMF-treated animals (Table 1).

Comparative observation across the photomicrographs of the hepatocytes of the animals showed well-outlined arrays of hepatic cells and vessels with no observable cytoarchitectural distortion in the control group (Fig. 2A), while cellular damage characterized with hemorrhage, fibrosis, and necrosis was observed in the hepatic cytoarchitecture of the PMF-treated rats (Fig. 2B). The pericardium, myocardium, and endocardium were

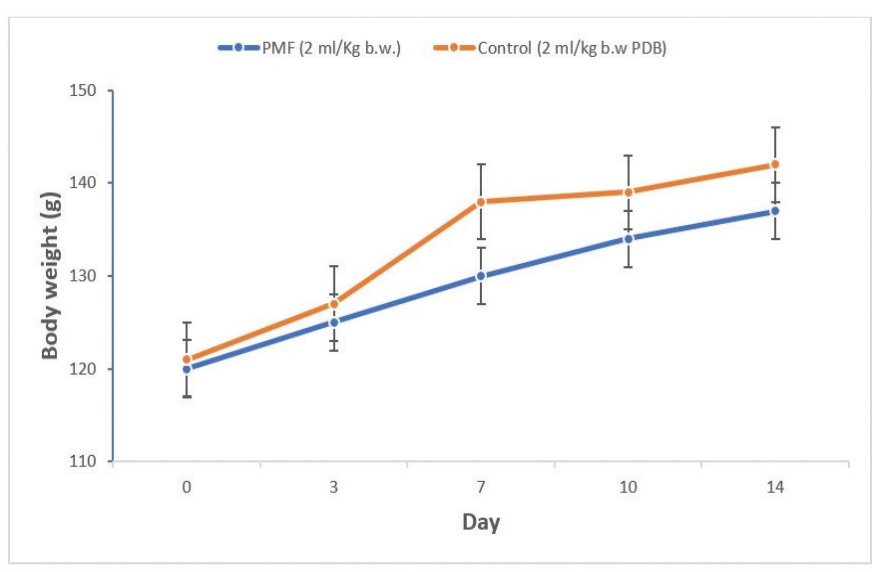

Figure 1. Body weight changes of the Wistar rats for the acute treatment with $\operatorname{PMF}(n=5$, mean \pm SEM $)$. conspicuous in the cardiocytes of the rats in the control group (Fig. 3A) compared to the degenerative changes with mild fibrosis observed in the PMF-administered animals (Fig. 3B). Similarly, no deleterious effects and cell distortion was observed in the cytoarchitecture of the kidney cells in the control group (Fig. 4A) which is in sharp contrast to the observed features in PMF-treated animals, where the kidney cells are characterized by overly large capsular spaces and hemorrhagic glomeruli which appeared to be edematous/dropsy (Fig. 4B).

\section{8-day toxicity testing}

No mortality, behavioral changes such as convulsion, tremor, diarrhea, and excess urine output were recorded during cage-side observations throughout the experimental period. The weight of both the PMF-treated rats and the control animals increased significantly $(p<0.05)$ throughout the experimental period with the highest percentage weight gain $(35.59 \%)$ observed in the rats administered $0.50 \mathrm{ml} \mathrm{PMF} \mathrm{(Table} \mathrm{2).} \mathrm{Except} \mathrm{for} \mathrm{the}$ observed significant differences $(p<0.05)$ in the WBC and PLT counts of the PMF-administered rats relative to the control, data obtained with respect to the hematological parameters of the PMFtreated animals are compared favorably with those of the control group (Table 3).

While the histoarchitecture of hepatocytes of the control group remained essentially well preserved (Fig. 5A), the 28-day repeated dosing with graded doses of PMF induced hepatocellular damage in the treated animals with obvious infiltrations including nuclear degeneration with significant cytotoxicity, necrosis, and distortion of the hepatic cytoarchitecture (Fig. 5B-D). Also, the cardiocytes of the control group were intact with adequately demonstrated striations across the myocardium (Fig. 6A). However, the cardiocytes of the rats administered with the lowest dose of PMF $(0.25 \mathrm{ml})$ showed degenerative changes with mild fibrosis (Fig. 6B), while those treated with $0.50 \mathrm{ml}$ of PMF revealed mild areas of vegetation which have eroded the normal orientation and structure of the heart cells (Fig. 6C). Fibrous tissues densely infiltrated by sparse inflammatory cells were also

Table 1. Effects of acute oral administration of PMF on hematological parameters of Wistar rats.

\begin{tabular}{ccc}
\hline Parameters & Control & P. microspora treated $(\mathbf{2} \mathbf{~ m l} / \mathbf{k g}$ b.w. $)$ \\
\hline WBC $\left(\times 10^{9} / \mathrm{l}\right)$ & $5.50 \pm 0.28$ & $7.67 \pm 0.26$ \\
NEUT $(\%)$ & $70.00 \pm 0.00$ & $68.67 \pm 0.88$ \\
LYMPH $(\%)$ & $28.67 \pm 0.91$ & $30.00 \pm 1.73$ \\
MONO $(\%)$ & $1.50 \pm 0.86$ & $1.00 \pm 0.57$ \\
RBC $\left(\times 10^{12} / 1\right)$ & $7.97 \pm 0.22$ & $8.67 \pm 0.62$ \\
HGB $(\mathrm{g} / \mathrm{dl})$ & $17.73 \pm 0.37$ & $18.90 \pm 1.09$ \\
PCV $(\%)$ & $27.67 \pm 0.88$ & $30.67 \pm 2.60$ \\
MCV $(\mathrm{Fl})$ & $69.00 \pm 1.73$ & $66.00 \pm 3.46$ \\
MCH $(\mathrm{Pg})$ & $19.15 \pm 0.11$ & $19.16 \pm 0.20$ \\
MCHC $(\mathrm{g} / \mathrm{dl})$ & $28.37 \pm 0.26$ & $28.86 \pm 1.12$ \\
PLT $\left(\times 10^{9} / 1\right)$ & $802.98 \pm 2.16$ & $802.45 \pm 8.95$ \\
\hline
\end{tabular}

$\mathrm{WBC}=$ white blood corpuscle; NEUT $=$ neutrophils; $\mathrm{LYMPH}=$ lymphocytes; $\mathrm{BASO}=$ basophils; $\mathrm{RBC}=$ red blood corpuscle; $\mathrm{HGB}=$ hemoglobin; $\mathrm{PCV}=$ packed cell volume; $\mathrm{MCV}=$ mean corpuscular volume; $\mathrm{MCH}=$ mean corpuscular hemoglobin; $\mathrm{MCHC}=$ mean corpuscular hemoglobin concentration; and PLT $=$ platelets $(n=5$, mean $\pm \mathrm{SEM})$ 


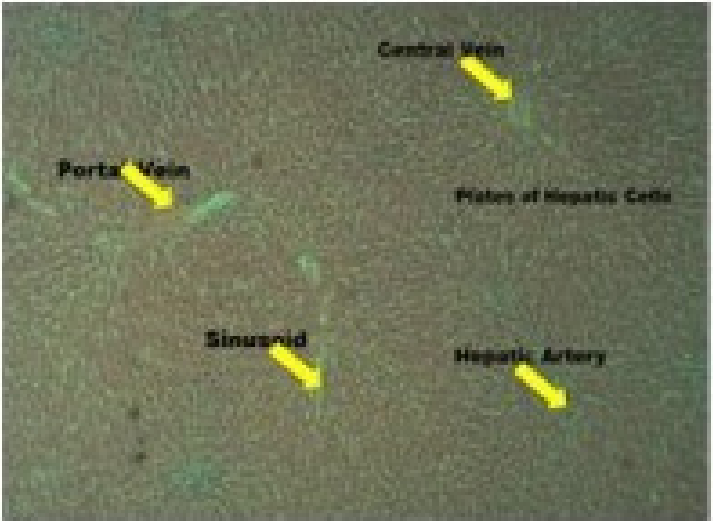

(A)

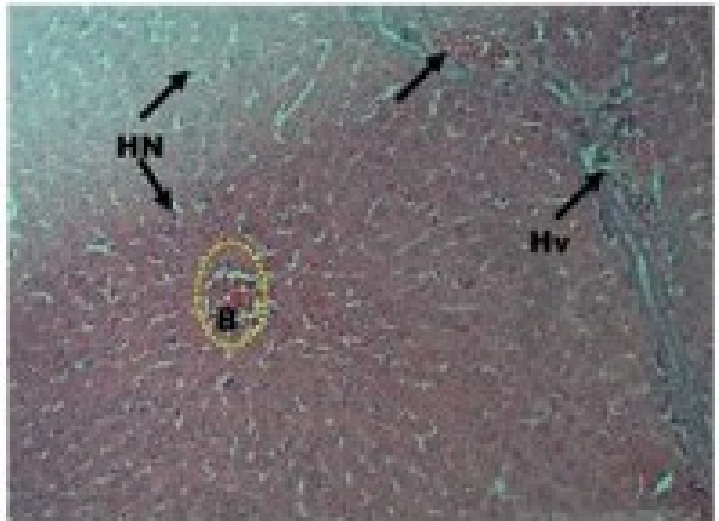

(B)

Figure 2. Photomicrograph (hematoxylin and eosin stained, $\mathrm{x} 10$ ) of hepatocyte of rats in the (A) control group showing well-arranged cells and vessels, without observable cytoarchitectural distortion, and (B) PMF-administered group showing round to oval-shaped hepatocytes with abundant granular and eosinophilic cytoplasm with scattered fats vacuoles, glycogen, and lipofuscin with characteristic pleomorphic to multiple nucleation. Hepatic vein: Hv, blood flow: B, and hepatic necrosis: HN.

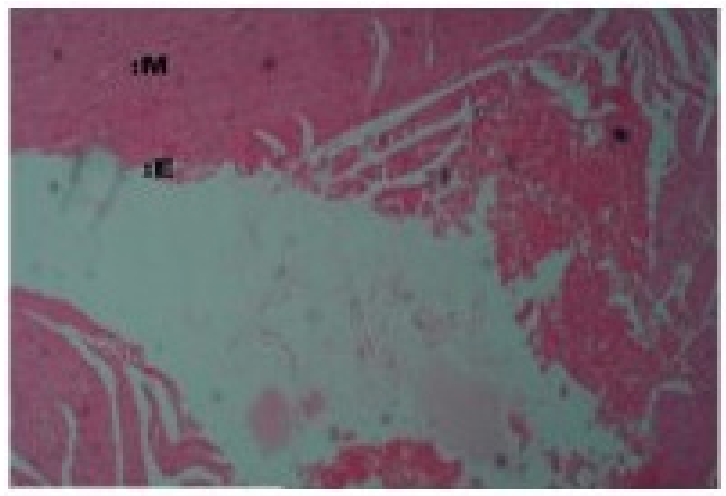

(A)

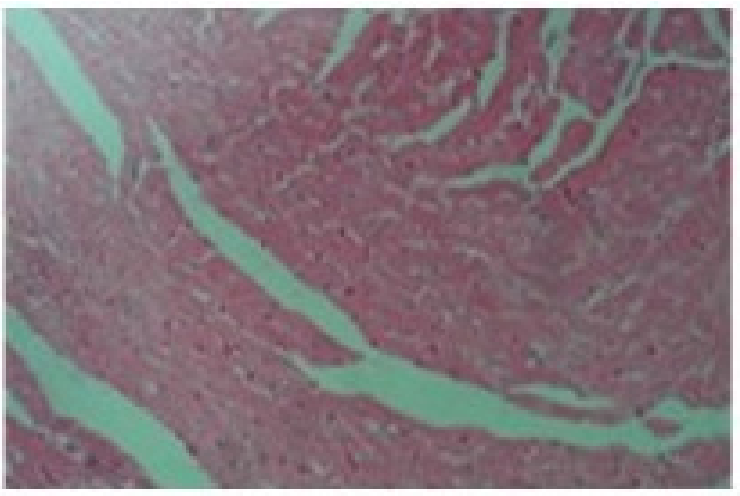

(B)

Figure 3. Photomicrographs (hematoxylin and eosin stained, $\mathrm{x} 10$ ) of cardiocytes of rats in the (A) control group showing well-demonstrated myocardium (M) and endocardium (E) as well as the intercalated disks and (B) PMF-treated animals showing well-demonstrated cardiocytes with intact striations across the myocardium.

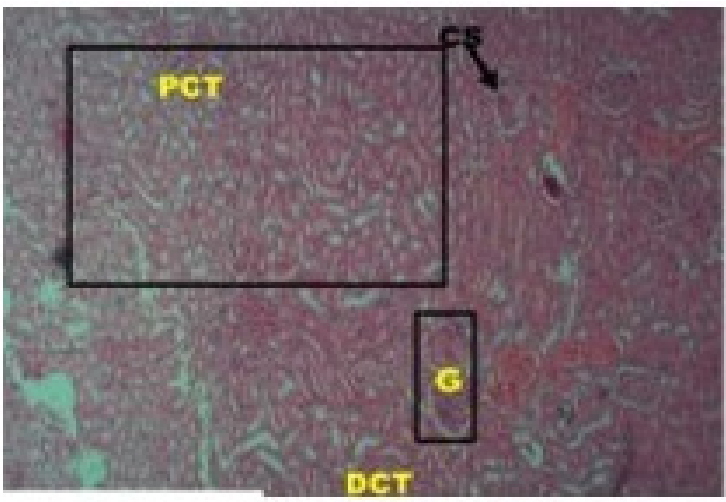

(A)

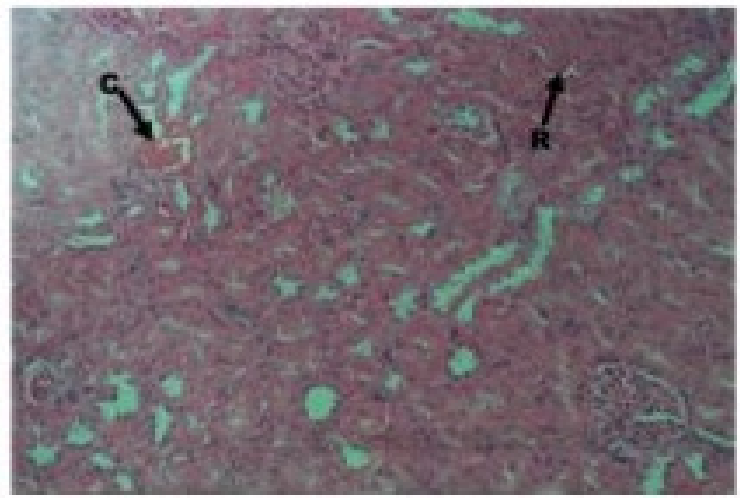

(B)

Figure 4. Photomicrographs (hematoxylin and eosin stained, $x 10)$ of renal corpuscles of rats in the (A) control group showing normal glomeruli (G) surrounded by capsular spaces (CS), proximal convoluted tubules (PCT), and distal convoluted tubules (DCT) without any distortion and (B) PMF-administered group showing mild degenerative changes $(\mathrm{R})$ characterized by overly large capsular spaces and hemorrhagic glomeruli (C) which appeared to be edematous and hemorrhagic. 
Table 2. Body weight changes (g) of the Wistar rats following 28-day treatment with PMF.

\begin{tabular}{ccccccc}
\hline & \multicolumn{7}{c}{ Week } \\
\hline Groups & 0 (initial) & 1st & 2nd & 3rd & 4th & Weight gain (\%) \\
$0.25 \mathrm{ml}$ & $128.30 \pm 12.80$ & $138.67 \pm 12.70$ & $136.33 \pm 9.56$ & $143.12 \pm 8.68$ & $162.67 \pm 4.76$ & 26.78 \\
$0.50 \mathrm{ml}$ & $118.12 \pm 5.77$ & $132.20 \pm 4.93$ & $154.00 \pm 1.52$ & $163.50 \pm 1.52$ & $160.00 \pm 8.50$ & 35.46 \\
$0.75 \mathrm{ml}$ & $110.33 \pm 11.53$ & $121.13 \pm 1.14$ & $132.54 \pm 9.08$ & $131.21 \pm 2.00$ & $130.25 \pm 1.00$ & 18.05 \\
Control & $130.00 \pm 5.08$ & $137.33 \pm 1.20$ & $148.44 \pm 7.20$ & $159.66 \pm 1.48$ & $148.11 \pm 2.41$ & 13.93 \\
\hline
\end{tabular}

$n=5$, mean \pm SEM.

Table 3. Effect of PMF on hematological parameters of Wistar rats following 28-day oral administration.

\begin{tabular}{|c|c|c|c|c|}
\hline \multirow[t]{2}{*}{ Parameters } & \multicolumn{4}{|c|}{ Treatments } \\
\hline & Control & $0.25 \mathrm{ml}$ PMF & $0.50 \mathrm{ml}$ PMF & $0.75 \mathrm{ml}$ PMF \\
\hline WBC $\left(\times 10^{9} / 1\right)$ & $9.87 \pm 0.95^{\mathrm{a}}$ & $5.17 \pm 0.43^{b}$ & $5.90 \pm 0.05^{\mathrm{b}}$ & $6.30 \pm 0.62^{b}$ \\
\hline NEUT (\%) & $56.00 \pm 8.66$ & $66.00 \pm 2.30$ & $67.67 \pm 0.33$ & $58.67 \pm 4.33$ \\
\hline LYMPH (\%) & $43.00 \pm 8.08$ & $32.60 \pm 2.03$ & $31.00 \pm 0.00$ & $40.00 \pm 4.62$ \\
\hline MONO (\%) & $1.00 \pm 0.58$ & $1.50 \pm 0.29$ & $1.50 \pm 0.29$ & $1.50 \pm 0.28$ \\
\hline $\mathrm{RBC}\left(\times 10^{12} / 1\right)$ & $6.94 \pm 0.32$ & $7.18 \pm 0.22$ & $6.67 \pm 0.34$ & $6.70 \pm 0.52$ \\
\hline $\operatorname{HGB}(\mathrm{g} / \mathrm{dl})$ & $18.00 \pm 0.34$ & $18.50 \pm 0.34$ & $16.60 \pm 0.23$ & $18.00 \pm 0.69$ \\
\hline PCV (\%) & $30.67 \pm 0.88$ & $32.00 \pm 1.15$ & $30.67 \pm 0.33$ & $30.67 \pm 2.02$ \\
\hline $\mathrm{MCV}(\mathrm{Fl})$ & $62.00 \pm 2.30$ & $62.00 \pm 0.57$ & $62.00 \pm 1.15$ & $65.00 \pm 2.89$ \\
\hline $\mathrm{MCH}(\mathrm{Pg})$ & $18.36 \pm 0.49$ & $18.37 \pm 0.89$ & $17.50 \pm 0.05$ & $19.40 \pm 0.69$ \\
\hline $\mathrm{MCHC}(\mathrm{g} / \mathrm{dl})$ & $29.19 \pm 0.22$ & $29.67 \pm 0.03$ & $28.17 \pm 0.49$ & $29.47 \pm 0.31$ \\
\hline PLT $\left(\times 10^{9} / 1\right)$ & $650.75 \pm 2.05^{\mathrm{a}}$ & $800.06 \pm 3.46^{\mathrm{b}}$ & $700.90 \pm 6.92^{b}$ & $800.06 \pm 6.64^{b}$ \\
\hline
\end{tabular}

$\mathrm{WBC}=$ white blood corpuscle; NEUT $=$ neutrophils; $\mathrm{LYMPH}=$ lymphocytes; $\mathrm{BASO}=$ basophils; $\mathrm{RBC}=$ red blood corpuscle; $\mathrm{HGB}=$ hemoglobin; $\mathrm{PCV}=$ packed cell volume; $\mathrm{MCV}=$ mean corpuscular volume; $\mathrm{MCH}=$ mean corpuscular hemoglobin; $\mathrm{MCHC}=$ mean corpuscular hemoglobin concentration; and PLT $=$ platelets $(n=5$, mean \pm SEM).

${ }^{a, b}$ Values carrying different superscript across the same row are significantly different $(p<0.05)$.

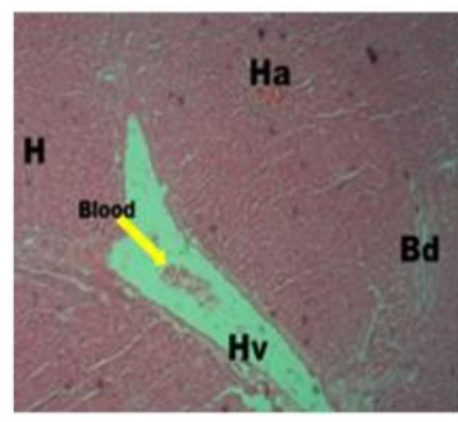

(A)

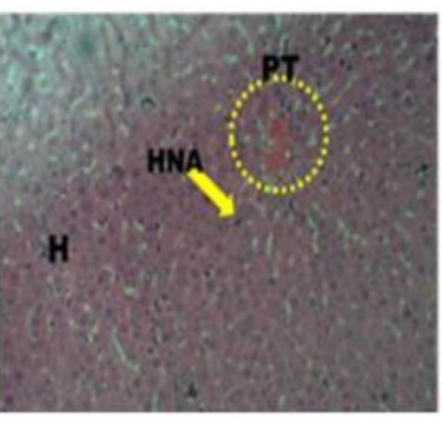

(B)

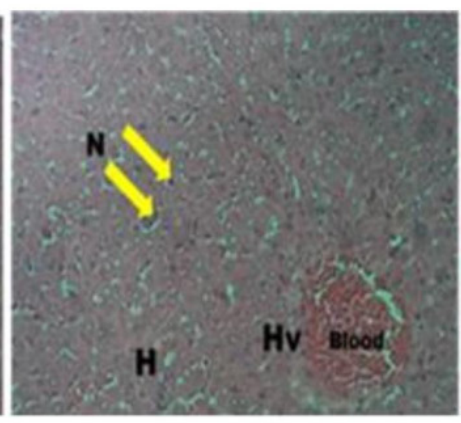

(C)

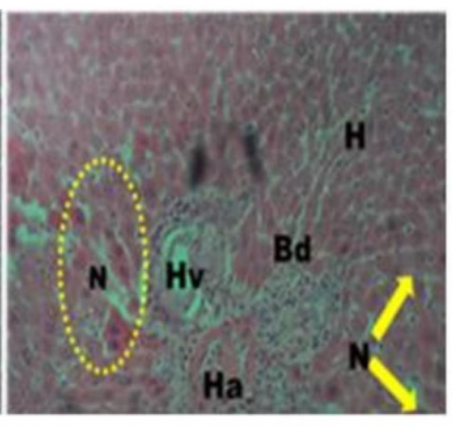

(D)

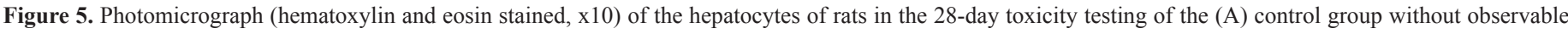

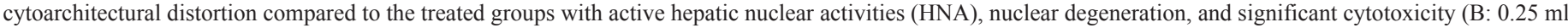

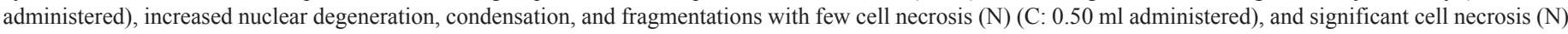

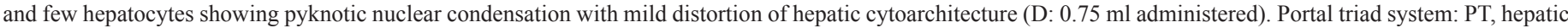
vein: Hv, hepatic artery: Ha, bile duct: Bd, and hepatocytes: $\mathrm{H}$.

observed (Fig. 6C). Furthermore, large vascular spaces some of which contained blood lining by epithelial cells were observed in the group administered with the $0.75 \mathrm{ml}$ PMF (Fig. 6D). Similarly, the repeated administration of the graded doses of PMF stimulated degeneration in the kidney cells with invasion by neutrophil and deposition of fibrin. The cortical tubules were shrunk and atrophic, and heavy polymorphonuclear infiltration was also observed around the renal artery (Fig. 7B-D). However, the animals in the control group were devoid of these observations as their renal cells revealed no observable histoarchitectural infiltrations (Fig. 7A).

\section{DISCUSSION}

For years, endophytes and mushrooms have been recognized as an important source of medicines and valuable 


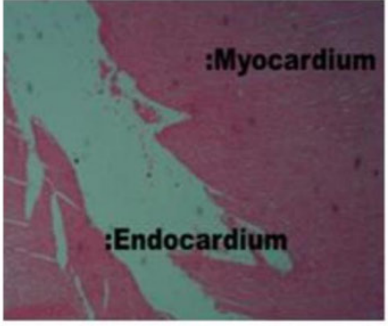

(A)

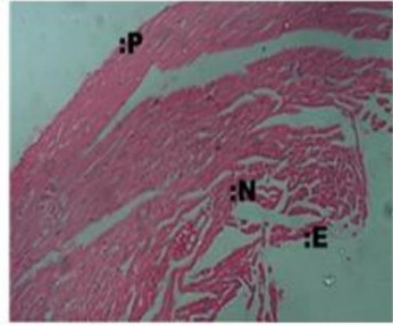

(B)

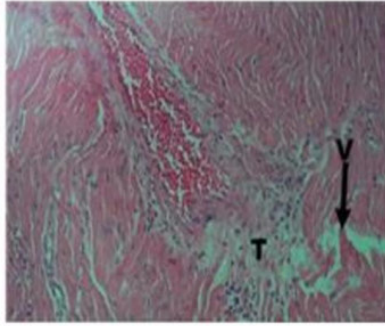

(C)

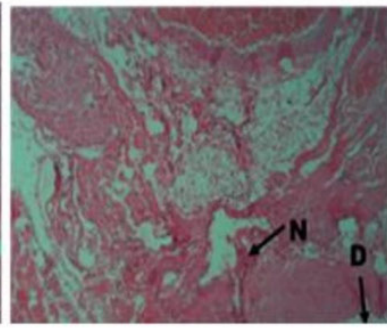

(D)

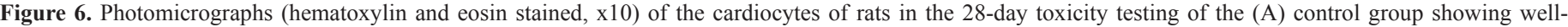

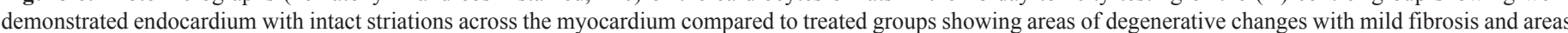

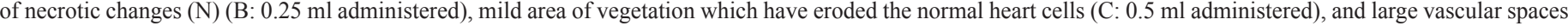

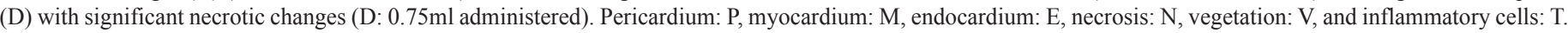

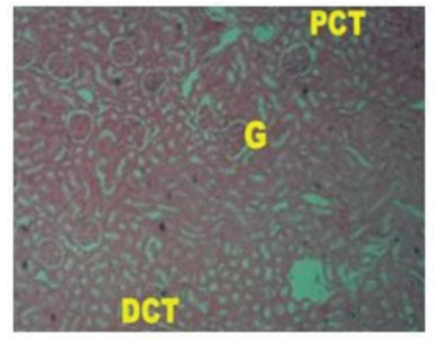

(A)

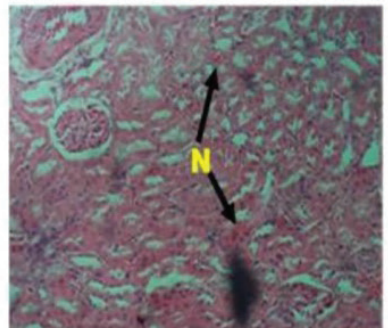

(B)

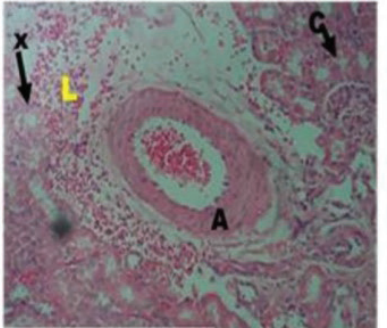

(C)

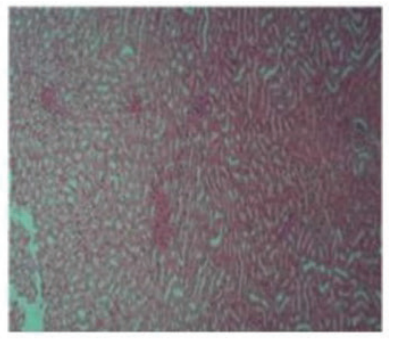

(D)

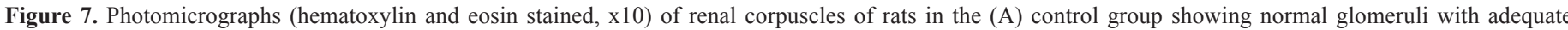

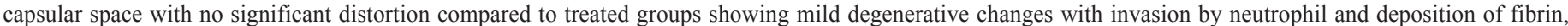

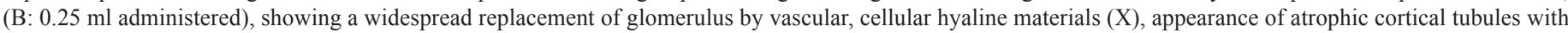

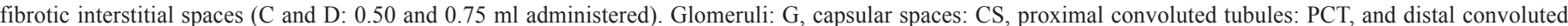
tubules: DCT.

raw materials in the food, cosmetic, and biochemical industries (Sharma et al., 2016; Tiwari, 2015). Despite these immense applications, endophytes remain hugely underutilized with their full benefits untapped. One of the reasons for the underutilization, especially as medicines could be adduced to absence or inadequate toxicological data to establish their safety profiles. While data exist on the characterization of novel compounds of therapeutic significance from endophytes (Alvin et al., 2014; Bhardwaj and Agrawal, 2014; Garuba et al., 2018; Gouda et al., 2016), studies are just emerging on their toxicological dynamics when consumed by livestock and humans. In this study, the nature of the toxicological effect of $P$. microspora on vital metabolic organs was investigated in vivo.

Although the acute toxicity evaluation is normally an initial phase in the determination of $\mathrm{LD}_{50}$ of a substance (Lorke, 1983), the observations in this study have suggested a probable no-observed-adverse-effect level of the $2 \mathrm{ml} / \mathrm{kg} \mathrm{b.w}$. dose of PMF at which the animals are expected to survive during a 14day observation period. However, while this observation was further corroborated by the nontoxic effect of the culture filtrate on the body weight and hematological parameters of the animals, the observed PMF-induced alterations and cytoarchitectural infiltrations in the hepatocytes, cardiocytes, and kidney cells of the treated rats are suggestive of selective toxicity of PMF on the investigated organs at the tested dose.

With the observed selective toxicity elicited by the $2 \mathrm{ml} /$ $\mathrm{kg}$ b.w. PMF acutely, a further probe was made through the repeated dosing toxicity testing of the filtrate at lower doses $(0.25-0.75 \mathrm{ml} /$ $\mathrm{kg}$ b.w.) to better comprehend the pattern of the observed effect. Interestingly, the nature of the selective toxicity became more evident from the significantly increased body weight of the PMFtreated rats relative to the control and this could be attributed to PMF-induced weight hypertrophy. Changes in body weight index constitute one of the initial vital signs of toxicity and could be a useful indicator of the health status of animals (Sireatawong et al., 2008). Although PMF has been reportedly rich in compounds of therapeutic importance, the observation in this study suggests that the repeated administration of the filtrate at the tested doses could have impaired normal metabolism and facilitated abnormal growth and developmental mechanisms in the animals. Changes in body weight of animals resulting from treatment with culture filtrate of fungi species have also been similarly reported in previous studies (Kwack et al., 2009; Park et al., 1999).

Evaluation of hematological indices can be employed to establish both the blood-related functions of a substance such as fungi culture filtrate or the degree of alteration or toxicological 
effect it has on the blood composition of animals (Sharma et al., 1983). Again, a type of parameter-specific toxicity nature of PMF was demonstrated by specifically altering the blood levels of PLTs and WBCs with no significant effect on other hematological parameters investigated in this study following 28-day exposure period. The blood level of WBC could be indicative of the defensive capability of an organism against pathogens or foreign substances (Ajani et al., 2014). In this study, the significantly increased WBCs of the PMF-administered rats could be indicative of inherent allergy, stress, inflammation, and infection attributable to PMF administration. Furthermore, the repeated dosing with PMF significantly and selectively increased PLT counts in the treated rats may be suggestive of its effect on thrombopoietin which could have impacted thrombopoiesis, enhanced vascular damage, and thrombocytopenia in animals. The observations on the WBCs and PLTs in this study are consistent with the reports of Fodor et al. (2006), Kwack et al. (2009), and Tikare et al. (2016), on the parameters-related effects of fungi isolates on the hematopoietic systems of experimental animals.

Histopathological examination is a major consideration that could be used to assess the nature of toxicity of an agent on vital metabolic organs and the results are usually complementary to the results of hematological analysis (Sabiu et al., 2015). The characteristic fibrosis, necrosis, and hemorrhagic cytoarchitectural infiltrations observed in the hepatocytes, heart, and kidney cells of the PMF-treated animals is an attestation to the organ damage effect of the repeated dosing with PMF at the investigated doses. With this observation, the vital roles of these metabolic organs including those of the liver in detoxification, deamination, nutrient supply, and indeed homeostasis are prone to compromise and could result in eventual organ and system failure. This submission is not only supportive of the nature of organ damage induced by the acute dose of the culture filtrate but also a further attestation to the pattern of selective toxicity exhibited by PMF in this study. In addition to the doses investigated and the experimental period, the nature of toxicity elicited by PMF may be adduced to its toxins or antinutrients, which might have coexisted with its acclaimed therapeutic compounds. The findings on the histopathological analyses of the organs in this study agree with previous studies (Fodor et al., 2006; Sharma et al., 1983; Thikare et al., 2011; Tikare et al., 2016), where observed infiltrations and cytopathic effects on the key organs of laboratory animals were attributed to treatment with fungi isolates.

\section{CONCLUSION}

Consequent to the data presented in this study, PMF exhibited parameter-specific toxicity on the hematopoietic system with a well-defined pattern of organ damage in the experimental animals. While this study has provided baseline information on the nature of liver, heart, and kidney damage by PMF at the investigated doses and exposure periods in vivo, studies seeking to confirm the presence of toxins/antinutritional factors and establishing comprehensive pharmacokinetic and pharmacodynamic data about PMF are essential. In this direction, efforts are underway.

\section{AUTHOR CONTRIBUTIONS}

All authors made substantial contributions to conception and design, acquisition of data, or analysis and interpretation of data; took part in drafting the article or revising it critically for important intellectual content; agreed to submit to the current journal; gave final approval of the version to be published; and agree to be accountable for all aspects of the work. All the authors are eligible to be an author as per the international committee of medical journal editors (ICMJE) requirements/guidelines.

\section{FUNDING}

There is no funding to report.

\section{CONFLICTS OF INTEREST}

The authors report no financial or any other conflicts of interest in this work.

\section{ETHICAL APPROVALS}

The study was reviewed and approved (UERC/ ASN/2019/1878) by the Animal Ethical Committee of the University of Ilorin, Ilorin, Nigeria.

\section{PUBLISHER'S NOTE}

This journal remains neutral with regard to jurisdictional claims in published institutional affiliation.

\section{REFERENCES}

Ajani EO, Sabiu S, Bamisaye FA, Ibrahim S, Salau BA. Evaluation of the acute and sub-acute toxicity effect of ethanolic leaves extract of Lagenaria breviflora (Bitter gourd) on hepatic and renal function of rats. J Pharm Biol Sci, 2014; 9:061-8.

Alvin A, Kristin I, Miller B, Neilan A. Exploring the potential of endophytes from medicinal plants as sources of antimycobacterial compounds. Microbiol Res, 2014; 169: 483-95.

Bhardwaj A, Agrawal P. Review fungal endophytes: as a storehouse of bioactive compound. World J Pharm Sci, 2014; 3:228-37.

Bing QH, Edward CY. Chemical and physical fixation of cells and tissues: an overview. In: Yeung ECT, Sumner MJ, Huang BQ, Stasolla C (eds.). Plant microtechniques and protocols. Springer International Publishing, Cham, Switzerland, 2015..

Chin YW, Balunas MJ, Chai HB, Kinghorn AD. Drug discovery from natural sources. AAPS J, 2006; 8(2):E239-253.

Deshmukh SK, Prakash V, Ranjan, N. Recent advances in the discovery of bioactive metabolites from Pestalotiopsis. Phytochem Rev, 2017; 16:883-920.

Elliot ML. Pestalotiopsis (Pestalotia) diseases of palm UIF/IFAS extension, University of Florida, Gainesville, Florida, 2018. Available via http:// edis.ifas.ufl.edu/pdffiles/PP/PP14100.pdf (Accessed 05 April 2019)2018.

Fodor J, Meyer K, Riedlberger M, Bauer J, Posa R. The distribution and elimination of fumonisins after oral administration of Fusarium verticillioides fungal culture. Magy Allatorvosok Lapja, 2006; 128:334-42.

Garuba T, Aare IO, Abdulrahaman AA, Aliyu MK, Amadi JE. Effects of fungal filtrates relative to soaking periods on seed germination of maize (Zea mays L.Poaceae). Biol Environ Sci J Trop, 2013; 10(4):061-4.

Garuba T, Sabiu, S, Lateef, AA, Adekanmbi AT, Adekanmbi MK. In-vitro cytotoxicity and antioxidant activities of Pestalotiopsis microspora culture filtrate. Malays J Appl Sci, 2018; 3(1):024-33.

Gouda S, Das G, Sen SK, Shin HS, Patra JK. Endophytes: a treasure house of bioactive compounds. Front Microbiol, 2016; 7:1538.

Jalander V, Gachande BD. Effect of fungal metabolites of some rhizosphere soil fungi on seed germination and seedling growth of some pulses and cereals. Sci Res Rep, 2012; 2(3):265-7.

Joseph B, Priya RM. Bioactive compounds from endophytes and their potential in pharmaceutical effect: a review. Am J Biochem Mol Biol, 2011; 1:291-309. 
Kwack SJ, Kim KB, Lee BM. Toxicological evaluation of an apicidin derivative, histone deacetylase inhibitor SD-2007 in mice. Arch Pharm Res, 2009; 32(5):789-97.

Lorke D. A new approach to practical acute toxicity testing. Ach Toxicol, $1983 ; 4: 275-87$.

Maharachchikumbura SSN, Guo LD, Chukeatirote E, Bahkali AH, Hyde KD. Pestalotiopsis-morphology, phylogeny, biochemistry and diversity. Fungal Divers, 2011; 50:167-87.

Metz AM, Haddad A, Worapong J, Long DM, Ford EJ, Hess WM, Strobel GA. Induction of the sexual stage of Pestalotiopsis microspora, a taxol-producing fungus. Microbiol, 2000; 146:2079-89.

Nagata N, Ando Y. Oxysporone, a phytotoxin isolated from the tea gray blight fungus Pestolatiopsis longiseta. Agric Biol Chem, 1989; $53: 2811$.

National Research Council (NRC). Guide for the care and use of laboratory animals. 8th edition, National Research Council, Washington, DC, 2011..

Olumese FE, Oboh HA. Antioxidant and antioxidant capacity of raw and processed Nigerian Beetroot (Beta vulgaris). Niger J Basic Appl Sci, 2016; 24(1):35-40.

Organization of Economic Co-operation and Development (OECD). Guideline for testing of chemicals, acute oral toxicity- acute toxicity class method. Organization of Economic Co-operation and Development (OECD), Paris, France, p 423, 2001.

Organization of Economic Co-operation and Development (OECD). Report of the validation of the updated test guideline 407: repeat dose 28-day oral toxicity study in laboratory rats. Series on testing and assessment No 59, ENV/JM/MONO, Organization of Economic Cooperation and Development (OECD), Paris, France, p 26, 2006.

Park JS, Lee KR, Kim JC, Lim SH, Seo JA, Lee YW. A haemorrhagic factor (apicidin) produced by toxic Fusarium isolates from soybean seeds. Appl Environ Microbiol, 1999; 4:126-30.

Radhia A, Hanen, N, Abdelkarim BA, Mohamed N. Phytochemical screening, antioxidant and antimicrobial activities of Erodium glaucophyllum (L.) L' Hérit. J Biomed Sci, 2018; 7(13):001-7.

Sabiu S, Ajani EO, Abubakar AA, Sulyman AO, Nurain IO, Irondi EA, Abubakar AY, Quadri DF. Toxicological evaluations of Stigma maydis aqueous extract on hematological and lipid parameters of Wistar rats. Tox Rep, $2015 ; 2: 638-44$.

Sharma D, Pramanik A, Agrawal PK. Evaluation of bioactive secondary metabolites from endophytic fungus Pestalotiopsis neglecta
BAB-5510 isolated from leaves of Cupressus torulosa D.Don. Biotech, 2016; 6(2):210

Sharma RB, Kumar A, Roy AN. Nature of hepatic damage induced by culture filtrates of seed-borne fungi in the laboratory rat. Toxicol Lett, 1983; 19:001-2.

Sireatawong S, Lertprasertsuke N, Srisawat U. Acute and subchronic toxicity study of the water extract from Tiliacora trianora (Colebr.) Diels in rats. Songklankarin J Sci Technol, 2008; 30:729-37.

Thikare V, Shridhar NB, Narayanaswamy HD, Isloor S, Jayakumar K. Sub chronic toxicity study of Rhizopus oryzae culture filtrate from groundnut hay in rats. Ind J Anim Res, 2011; 45(2):130-4.

Tikare VP, Shridhar NB, Jagadeesh S, Chitra BM, Narayanaswamy HD. Molecular characterization and subchronic toxicity study of Fusarium sporotrichoides fungus in rats isolated from groundnut hay. Int J Sci Environ Tech, 2016; 5(4): 2135-2141

Tiwari K. The future products: endophytic fungal metabolites. J Biodiv Bioprosp Dev, 2015; 2:001-7.

Venkatasubbaiah P, van Dyke CG, Chilton WS. Phytotoxins produced by Pestalotiopsis oenotherae, a pathogen of evening primrose. Phytochem, 1991; 30:1471-4.

Vinay PT, Shridhar NB, Sanganal JS, Narayanaswamy HD, Isloor S, Chitra BM. Multimycotoxin analysis using LC-MS/MS and sub chronic toxicity study of Fusarium semitectum fungus isolated from groundnut hay. J Cell Tissue Res, 2014; 14(2):4377-84.

Wei JG, Xu T. Pestalotiopsis kunmingensis sp. nov., an endophyte from Podocarpusmacrophyllus. Fungal Divers, 2004; 15 247-54.

World Health Organization. Basic OECD principles of GLP World Health Organization, Geneva, Switzerland, 1998.

How to cite this article:

Garuba T, Lateef AA, Atunwa SA, Lateef AY, Kolawole OO, Sabiu S. Nature of hematopoietic system alteration and organ damage induced by fungal filtrate of Pestalotiopsis microspora in vivo. J Appl Pharm Sci, 2021; 11(03):084-091. 